Research Article

\title{
DYNAMIC ANALYSIS OF UNIFORM AND NON-UNIFORM CROSS-SECTION CANTILEVER SANDWICH BEAMS
}

\begin{abstract}
Mesut HÜSEYINOĞLU ${ }^{1^{*}}$, Murat ŞEN ${ }^{2}$, Osman YİĞİD ${ }^{3}$, Orhan ÇAKAR
In this study, an analytical solution for dynamic analysis of uniform and nonuniform cross-section cantilever sandwich beam is presented. The sandwich beam was assumed to be an Euler-Bernoulli beam and formed with a thin core and two thin skin layers. So the shear deformations and rotational effects were neglected. The equivalent flexural rigidity was obtained for the entire sandwich structure. Some implementations for the solution method are given and the results are compared with numerical solutions. The usability of the Euler-Bernoulli Beam Theory for thin layered uniform or non-uniform sandwich beams is investigated. The solutions obtained from analytical and numerical solutions are in good agreement.
\end{abstract}

Key words: Natural Frequency, Sandwich Beam, Mode Shape, Vibration

\section{Introduction}

Sandwich structures have a very wide usage from aircrafts to automotive, marine, storage and transportation. Due to their light weight, high bending stiffness and damping capability, they are preferred in many engineering applications. Generally a sandwich structure is combined with two elastic thin skin layers and a viscoelastic core layer. Using the advantages of light weight, vibration isolation and flexural strength properties of these structures come forward in mechanical and structural engineering. These advantages make researchers focus on these structures and a lot of papers have been published on this topic for the last decade. Some of them are the studies of analytical approaches when the big amount of them are numerical and experimental analysis. Khalili et al. [1] investigated the free vibrations of three layer sandwich beams by using dynamic stiffness method considering different density, thickness and shear modulus of core materials for various boundary conditions. Rabinovitch and Hamed [2] made a study on dynamic behavior of sandwich beams with viscoelastic soft core. They took into account the shear and transverse effects for sandwich beams by utilizing the Maxwell and Kelvin-Voigt models for viscoelasticity. A model was formulated by Palmeri and Ntotsios [3] for dynamic analysis of sandwich beams by using mathematical derivation of generalized Maxwell model of shear type viscoelastic layer for the core and consisting of two parallel Euler-Bernoulli elastic beams. Irazu et al. [4] made a study on the sandwich structures with viscoelastic adhesive films. They

\footnotetext{
${ }^{1}$ Department of Mechanical Engineering, Dicle University, Diyarbakır, Turkey, (mesuth@dicle.edu.tr) (Dhttps://orcid.org/00000002-6130-6658

${ }^{2}$ Department of Mechanical Engineering, Furat University, Elazı̆g, Turkey, (msen@ firat.edu.tr) $@$ https://orcid.org/0000-0002-30635635

${ }^{3}$ Department of Mechanical Engineering, Furat University, Elazı̆g, Turkey, (oyigid@ firat.edu.tr) ${ }^{\circledR}$ https://orcid.org/0000-0002-17981250 $6947-3875$

${ }^{4}$ Department of Mechanical Engineering, Firat University, Elazığ, Turkey, (cakaro@firat.edu.tr) (D) https://orcid.org/0000-0001-
} 
characterized the sandwich structure and the viscoelastic films by using standard experimental dynamic tests by obtaining loss factor, storage modulus and shear complex modulus. A method was presented by Sakiyama et al. [5] so as to analysis the vibration characteristics of a three layered sandwich structure combined with elastic or viscoelastic core by using an approach utilizing the solution of differential equations of the flexural behavior of the structure under loading condition. Wang et al. [6] analyzed vibration characteristics of three layered sandwich plates with bounded piezoelectric patch actuators and a viscoelastic core. They handled the viscoelastic core as complex layer and variant with frequency and temperature by using the Golla-Hughes-McTavish method to account for the frequency dependent properties of it. Zghal et al. [7] gave an approach to deal with the local nonlinearities on the dynamic behavior of such model assembled structures.

Finite element solutions are widely used for sandwich structures [8]-[12]. Zhen and Wanji [13] used a high order zigzag method for vibration analysis of composite beams. They made an elimination for the higher order derivatives of displacement parameters of shear stresses by utilizing the variational principle of Hu-Washizu. Pham et al. [14] presented a shear deformable finite element formulation for free and forced vibration analysis of two directional functionally graded sandwich beams which combined with a homogeneous ceramic core layer and two functionally graded skin layers. High order methods are used successfully in many studies [15]-[17].

In sandwich structures, core material, pattern or cell type and geometry effect the dynamic characteristics of structures [18]-[22]. Sun and Thamburaj [23] studied on sound and vibration transmission across a sandwich beam combined with anisotropic layers. They investigated the effects of damping, thickness of the layers and material density on the sound transmission loss. They focused on selecting a core material and geometry to transfer the vibration from the top skin into the shear deformation and in-plane wave in the core for reducing the deflection of the bottom skin of the sandwich beams.

There are also number of analytical or numerical studies on various kinds of functionally graded structures [24]-[35]. Yang et al. [36] studied on the resonance frequencies and critical speed of an axially moving sandwich beam and on the relationship between the axially travelling speed and the natural frequencies. Lougou et al. [37] developed a double scale asymptotic method which seperates the original problem into two small ones to analyze the layered sandwich structures with viscoelastic core.

In this study, an analytical solution for free vibration characteristics of uniform and non-uniform cross-section sandwich beams for fixed-free boundary condition is presented. The sand wich beam was assumed to be a thin Euler- Bernoulli beam. So the shear deformations and the rotational effects were neglected. In the following sections, the methodology, the application and the conclusion parts are given respectively.

\section{Material and Method}

There are two common approaches in vibrations of beams one of which is Euler-Bernoulli beam theory and the second is Timoshenko beam theory. In the former approach the shear deformations and rotational effects are neglected while in the latter approach they are taken in account. Euler-Bernoulli beam theory is suitable for thin beams (the thickness of the beam is very small according to width and length of it). But for thick beams Timoshenko beam theory is more appropriate. In our study, the sandwich beam is considered thin enough to neglect the rotational effects. So, Euler-Bernoulli beam theory is used. Free body diagram of a small part of a simple Euler-Bernoulli beam is given in Figure 1. 

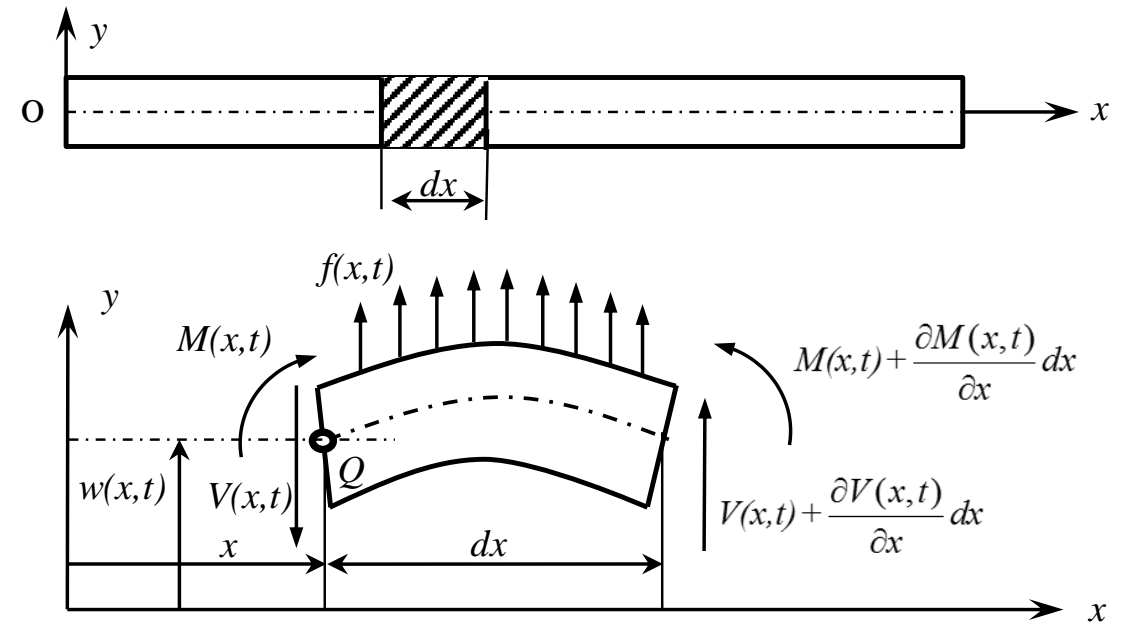

Figure 1. Free body diagram of a small part of a simple Euler-Bernoulli beam.

If a small unit element $d x$ is handled of an Euler-Bernoulli beam exposed to a distributed load $f(x, t)$, the differential equation of motion can be expressed as:

$$
f(x, t) d x+\left[V(x, t)+\frac{\partial V(x, t)}{\partial x} d x\right]-V(x, t)=m(x) d x \frac{\partial^{2} w(x, t)}{\partial t^{2}}
$$

Here, $m(x)$ is the linear mass density $(\mathrm{kg} / \mathrm{m})$. The right part of the equation is the inertial force of the small element. By neglecting the rotary inertia if the moments about $\mathrm{z}$ axis trough the point $Q$ are taken and after summation:

$$
[f(x, t) d x] \frac{d x}{2}+\left[V(x, t)+\frac{\partial V(x, t)}{\partial x} d x\right] d x+\left[M(x, t)+\frac{\partial M(x, t)}{\partial x} d x\right]-M(x, t)=0
$$

Equation (2) can be obtained and by simplifying it, the equation (3) can be written.

$$
\left[\frac{f(x, t)}{2}+\frac{\partial V(x, t)}{\partial x}\right](d x)^{2}+\left[V(x, t)+\frac{\partial M(x, t)}{\partial x}\right] d x=0
$$

Here, $d x$ is very small and $(d x)^{2}$ is almost zero, so the term $(d x)^{2}$ can be neglected. So, this expression can be rewritten as in equation (4):

$$
V(x, t)=-\frac{\partial M(x, t)}{\partial x}
$$

By substitution this expression into equation (1)

$$
f(x, t) d x-\frac{\partial^{2}}{\partial x^{2}}[M(x, t)] d x=m(x) d x \frac{\partial^{2} w(x, t)}{\partial t^{2}}
$$

Also, the relation between the bending moment and the deflection of beam can be expressed by 


$$
M(x, t)=E I(x) \frac{\partial^{2} w(x, t)}{\partial x^{2}}
$$

Here $E I(x)$ is the flexural rigidity and combined by modulus of elasticity $(E)$ of the material and moment of inertia $(I(x))$ of the beam changes through $x$ direction respectively. Substitution of equation (6) into (5) and to simplify with dividing the equation (5) by $d x$ yields:

$$
\frac{\partial^{2}}{\partial x^{2}}\left[E I(x) \frac{\partial^{2} w(x, t)}{\partial x^{2}}\right]+m(x) \frac{\partial^{2} w(x, t)}{\partial t^{2}}=f(x, t)
$$

The equivalent flexural rigidity $E I(x)$ can be written for $\mathrm{n}$ layers sandwich structure with combination of the layers can be expressed as:

$$
E I(x)=E_{1} I_{1}(x)+E_{2} I_{2}(x) \ldots \ldots \ldots E_{n} I_{n}(x)
$$

For three layer symmetric sandwich beam with two face sheets and a core layer, the equation (8) can be rewritten:

$$
E I(x)=2 E_{f} I_{f}(x)+E_{c} I_{c}(x)
$$

\subsection{For uniform cross-section sandwich beams}

For uniform cross-section sandwich beams, the linear mass density and the flextural rigidity are constant due to the uniform thickness and width through the length. A uniform cross-section sandwich beam is illustrated in Figure 2.

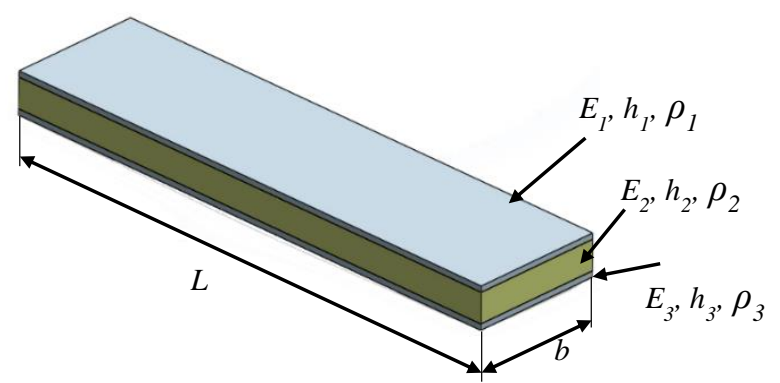

Figure 2. A uniform cross-section sandwich beam.

For uniform cross-section sandwich beams $(E I(x)$ and $m(x)$ are constant) the equation (7) can be written as:

$$
\frac{\partial^{2}}{\partial x^{2}}\left[E I \frac{\partial^{2} w(x, t)}{\partial x^{2}}\right]+m \frac{\partial^{2} w(x, t)}{\partial t^{2}}=f(x, t)
$$

For free vibrations (with no excitation force) and for uniform cross-section beams, simplified equation (11) is obtained, where, $a^{2}=E I / m$

$$
a^{2} \frac{\partial^{4} w(x, t)}{\partial x^{4}}+\frac{\partial^{2} w(x, t)}{\partial t^{2}}=0
$$


With the separation of variables the solution can be expressed by equalizing to a constant $\omega^{2}$,

$$
a^{2} \frac{X^{I V}(x)}{X(x)}=-\frac{\ddot{T}(t)}{T(t)}=\omega^{2} \quad\left(X^{I V}=\frac{d^{4} X}{d x^{4}}, \quad \ddot{T}=\frac{d^{2} T}{d t^{2}}\right)
$$

This equation can be decomposed spatial and time parts.

$$
\ddot{T}(t)+\omega^{2} T(t)=0 \quad T(t)=A \sin \omega t+B \cos \omega t
$$

Here, $A$ and $B$ can be determined by the initial conditions. By rearranging the equation (12) for spatial equation

$$
X^{I V}(x)-\frac{\omega^{2}}{a^{2}} X(x)=0
$$

By defining $x=A e^{\lambda x}$ and recalling the equation (11) yields:

$$
\beta^{4}=\frac{\omega^{2}}{a^{2}}=\frac{m \omega^{2}}{E I}
$$

Then general solution of the equation (14) can be calculated in the form as follows:

$$
X(x)=c_{1} \sin \beta x+c_{2} \cos \beta x+c_{3} \sinh \beta x+c_{4} \cosh \beta x
$$

Here $C_{1}, C_{1}, C_{1}, C_{1}$ are constant and one can obtain those constants from boundary conditions. The boundary conditions for cantilever beam can be written as follows:

For clamped end at $x=0$

$$
\begin{aligned}
& x(0)=0 \\
& x(0)=c_{1} \sin 0+c_{2} \cos 0+c_{3} \sinh 0+c_{4} \cosh 0=0 \\
& c_{4}=-c_{2} \\
& x^{\prime}(0)=0 \\
& x^{\prime}(0)=\lambda c_{1} \cos 0-\lambda c_{2} \sin 0+\lambda c_{3} \cosh 0+\lambda c_{4} \sinh 0=0 \\
& c_{3}=-c_{1}
\end{aligned}
$$

For free end at $x=L$

$$
\begin{aligned}
& x^{\prime \prime}(L)=0 \\
& x^{\prime \prime}(L)=-\beta^{2} c_{1} \sin \beta L-\beta^{2} c_{2} \cos \beta L+\beta^{2} c_{3} \sinh \beta L+\beta^{2} c_{4} \cosh \beta L=0 \\
& c_{1}(\sin \beta L+\sinh \beta L)+c_{2}(\cos \beta L+\cosh \beta L)=0 \\
& \quad x^{\prime \prime \prime}(L)=0 \\
& x^{\prime \prime \prime}(L)=-\beta^{3} c_{1} \cos \beta L+\beta^{3} c_{2} \sin \beta L+\beta^{3} c_{3} \cosh \beta L+\beta^{3} c_{4} \sinh \beta L=0 \\
& c_{1}(\cos \beta L+\cosh \beta L)+c_{2}(-\sin \beta L+\cosh \beta L)=0
\end{aligned}
$$

If the last two equalities are written in matrix form 


$$
\left[\begin{array}{cc}
(\sin \beta L+\sinh \beta L) & (\cos \beta L+\cosh \beta L) \\
(\cos \beta L+\cosh \beta L) & (-\sin \beta L+\sinh \beta L)
\end{array}\right]\left[\begin{array}{l}
c_{1} \\
c_{2}
\end{array}\right]=\left[\begin{array}{l}
0 \\
0
\end{array}\right]
$$

For nontrivial solution, the determinant of the first part of the left equation must be zero. For this:

$$
\cos \beta_{n} L \cosh \beta_{n} L+1=0 \quad n=1,2, \ldots \ldots . . n
$$

The characteristic equation can be obtained. The first four roots of equation (22) are calculated as: $\beta_{n} L=1.8751,4.6941,7.8548,10.9955 \quad(n=1,2,3,4)$

With a transformation of $\beta_{n} L=\lambda_{n}$ the natural frequencies equation can be obtained:

$$
\begin{gathered}
\omega_{n}=\frac{\lambda_{n}^{2}}{L^{2}} \sqrt{\frac{E I}{m}} \quad(\mathrm{r} / \mathrm{s}) \\
f_{n}=\frac{\lambda_{n}^{2}}{2 \pi L^{2}} \sqrt{\frac{E I}{m}}(\mathrm{~Hz}) \quad\left(\omega_{n}=2 \pi f_{n}\right)
\end{gathered}
$$

For a uniform cross-section three layered sandwich beam, the equivalent flexural rigidity and mass density can be expressed as:

$$
\begin{gathered}
E I=E_{1}\left[b \frac{h_{1}^{3}}{12}+b h_{1}\left(\frac{h_{1}+h_{2}}{2}\right)^{2}\right]+E_{2} b \frac{h_{2}^{3}}{12}+E_{3}\left[b \frac{h_{3}^{3}}{12}+b h_{3}\left(\frac{h_{3}+h_{2}}{2}\right)^{2}\right] \\
m=\rho_{1} A_{1}+\rho_{2} A_{2}+\rho_{3} A_{3}
\end{gathered}
$$

By using equations (17-22), the mode shapes can be obtained as in equation (27).

$$
X(x)=\left(c_{1}\right)_{n}\left[\frac{\left(\sin \beta_{n} L+\sinh \beta_{n} L\right)}{\left(\cos \beta_{n} L+\cosh \beta_{n} L\right)}\left(\cos \beta_{n} x-\cosh \beta_{n} x\right)+\sin \beta_{n} x+\sinh \beta_{n} x\right]
$$

\subsection{For non-uniform cross-section sandwich beams}

For non-uniform cross-section sandwich beams, the linear mass density and the flextural rigidity are not constant, because the width of the sandwich beam is not constant through the length while the length and the total height of the sandwich beam is constant. A non-uniform cross-section sandwich beam is illustrated in Figure 3. 


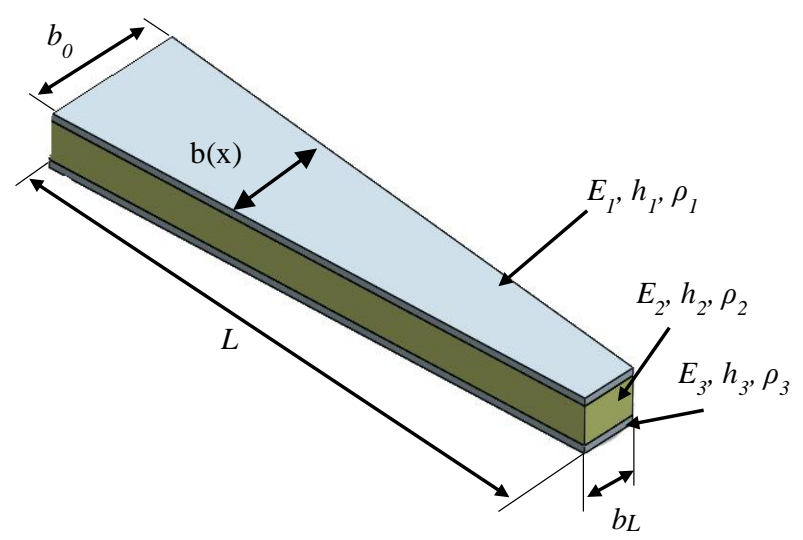

Figure 3. A non-uniform cross-section sandwich beam.

For non-uniform cross-section sandwich beams flextural rigidity $(E I(x))$ and linear mass density $(m(x))$ in equation (7) are not constant and they change through the length. Assuming the total height of the sandwich beam is constant and the width of the beam is changing exponentially through the length as:

$$
b(x)=b_{0} e^{-\delta x}
$$

where $b_{o}$ is the width of the beam at the fixed end and $\delta$ is the non-uniformity parameter. Mass density and the flexural rigidity changes with respect to $b(x)$.

$$
\begin{gathered}
m(x)=\left(\rho_{1} h_{1}+\rho_{2} h_{2}+\rho_{3} h_{3}\right) b(x) \\
E I(x)=b(x)\left\{E_{1}\left[\frac{h_{1}^{3}}{12}+h_{1}\left(\frac{h_{1}+h_{2}}{2}\right)^{2}\right]+E_{2} \frac{h_{2}^{3}}{12}+E_{3}\left[\frac{h_{3}^{3}}{12}+h_{3}\left(\frac{h_{3}+h_{2}}{2}\right)^{2}\right]\right\}
\end{gathered}
$$

Assuming $m_{0}=\left(\rho_{1} h_{1}+\rho_{2} h_{2}+\rho_{3} h_{3}\right)$ and

$$
I_{0}=\left\{E_{1}\left[\frac{h_{1}^{3}}{12}+h_{1}\left(\frac{h_{1}+h_{2}}{2}\right)^{2}\right]+E_{2} \frac{h_{2}^{3}}{12}+E_{3}\left[\frac{h_{3}^{3}}{12}+h_{3}\left(\frac{h_{3}+h_{2}}{2}\right)^{2}\right]\right\}
$$

the mass density and the flexural rigidity simplify as:

$$
\begin{aligned}
& m(x)=m_{0} b(x) \\
& E I(x)=I_{0} b(x)
\end{aligned}
$$

Assuming the solution of the equation (7) to be

$$
y(x, t)=y(x) \sin \omega t
$$


then after simplification, it reduces to

$$
\frac{d^{4} y}{d x^{4}}-2 \delta \frac{d^{3} y}{d x^{3}}+\delta^{2} \frac{d^{2} y}{d x^{2}}-\omega^{2} \frac{m_{0}}{I_{0}} y(x)=0
$$

Solution of Eq. (33) can be obtained as:

$$
y(x)=e^{\frac{\delta}{2} x}\left[C_{1} \cos (a x)+C_{2} \sin (a x)+C_{3} \cosh (b x)+C_{4} \sinh (b x)\right]
$$

where,

$$
a=\frac{\sqrt{4 \omega_{0}+\delta^{2}}}{2} ; \quad b=\frac{\sqrt{4 \omega_{0}-\delta^{2}}}{2}
$$

Here,

$$
\omega_{0}=\sqrt{\frac{m_{0}}{I_{0}} \omega^{2}}
$$

and $C_{1}, C_{2}, C_{3}, C_{4}$, are constant and can be determined from boundary conditions.

For fixed-free beam boundary conditions are:

$$
y(0)=0 ; \quad y^{\prime}(0)=0 ; \quad y^{\prime \prime}(L)=0 ; \quad y^{\prime \prime \prime}(L)=0
$$

The natural frequencies of the fixed-free beam can be obtained substituting these boundary conditions into Eq. (34). A characteristic equation for this configuration of beam is obtained as:

$$
\begin{aligned}
& \frac{a b}{8}-\frac{a b^{3}}{2}+\frac{a^{3} b}{2}+a b^{5}+a^{5} b+\cos (a L) \sinh (b L)\left(-\frac{a^{3}}{4}-\frac{a b^{2}}{4}+a b^{4}+a^{3} b^{2}\right) \\
& +\cos (a L) \cosh (b L)\left(-\frac{a b}{8}+\frac{a b^{3}}{2}-\frac{a^{3} b}{2}+2 a^{3} b^{3}\right) \\
& +\sin (a L) \sinh (b L)\left(-\frac{a^{2}}{16}+\frac{a^{4}}{4}+\frac{b^{2}}{16}+\frac{b^{4}}{4}+3 \frac{a^{2} b^{2}}{2}-a^{2} b^{4}+a^{4} b^{2}\right) \\
& +\sin (a L) \cosh (b L)\left(\frac{b^{3}}{4}+\frac{a^{2} b}{4}+a^{4} b+a^{2} b^{3}\right)=0
\end{aligned}
$$

\section{Applications}

In application studies, for both uniform and non-uniform cross-section sandwich beams the natural frequencies were obtained by using presented analytical method and by utilizing numerical approach. The non-uniformity is expressed as a function $\left(b(x)=b_{0} e^{-2 x}\right)$ for which the width changes through the length. The numerical studies were made by using ANSYS Wokbech finite element software. For both uniform and non-uniform sandwich beams, the material properties are given in Table 1. The sandwich structures are made by using two aluminum face layers and ABS core layer for both types. The layers of the sandwich beams were assumed to be thin enough as it is supposed to be for Euler-Bernoulli beam theory approach and assumed to remain in contact form with each other with perfect connection. 
Table 1. Material properties of uniform and non-uniform cross-section sandwich beams.

\begin{tabular}{ccc}
\hline Parameters & $\begin{array}{c}\text { Uniform } \\
\text { Cross-section }\end{array}$ & $\begin{array}{c}\text { Non-uniform } \\
\text { Cross-section }\end{array}$ \\
\hline$E_{1}(\mathrm{GPa})$ & 69 & 69 \\
$E_{2}(\mathrm{GPa})$ & 2 & 2 \\
$E_{3}(\mathrm{GPa})$ & 69 & 69 \\
$\rho_{1}\left(\mathrm{~kg} / \mathrm{m}^{3}\right)$ & 2700 & 2700 \\
$\rho_{2}\left(\mathrm{~kg} / \mathrm{m}^{3}\right)$ & 1020 & 1020 \\
$\rho_{3}\left(\mathrm{~kg} / \mathrm{m}^{3}\right)$ & 2700 & 2700 \\
$h_{1}(\mathrm{~mm})$ & 1 & 1 \\
$h_{2}(\mathrm{~mm})$ & 1 & 1 \\
$h_{3}(\mathrm{~mm})$ & 1 & 1 \\
$b(\mathrm{~mm})$ & $50($ constant $)$ & $b(x)=b_{0} e^{-2 x}$ \\
$L(\mathrm{~mm})$ & 500 & 500 \\
\hline
\end{tabular}

For a few bending modes, the natural frequencies obtained by presented analytical solution are illustrated in Figure 4. (in which the value of the characteristic equation is zero) and the ones obtained by numerical approach are given in Table 2 and Table 3 with percentage difference for both uniform and non-uniform cross-section sandwich beams comperatively.
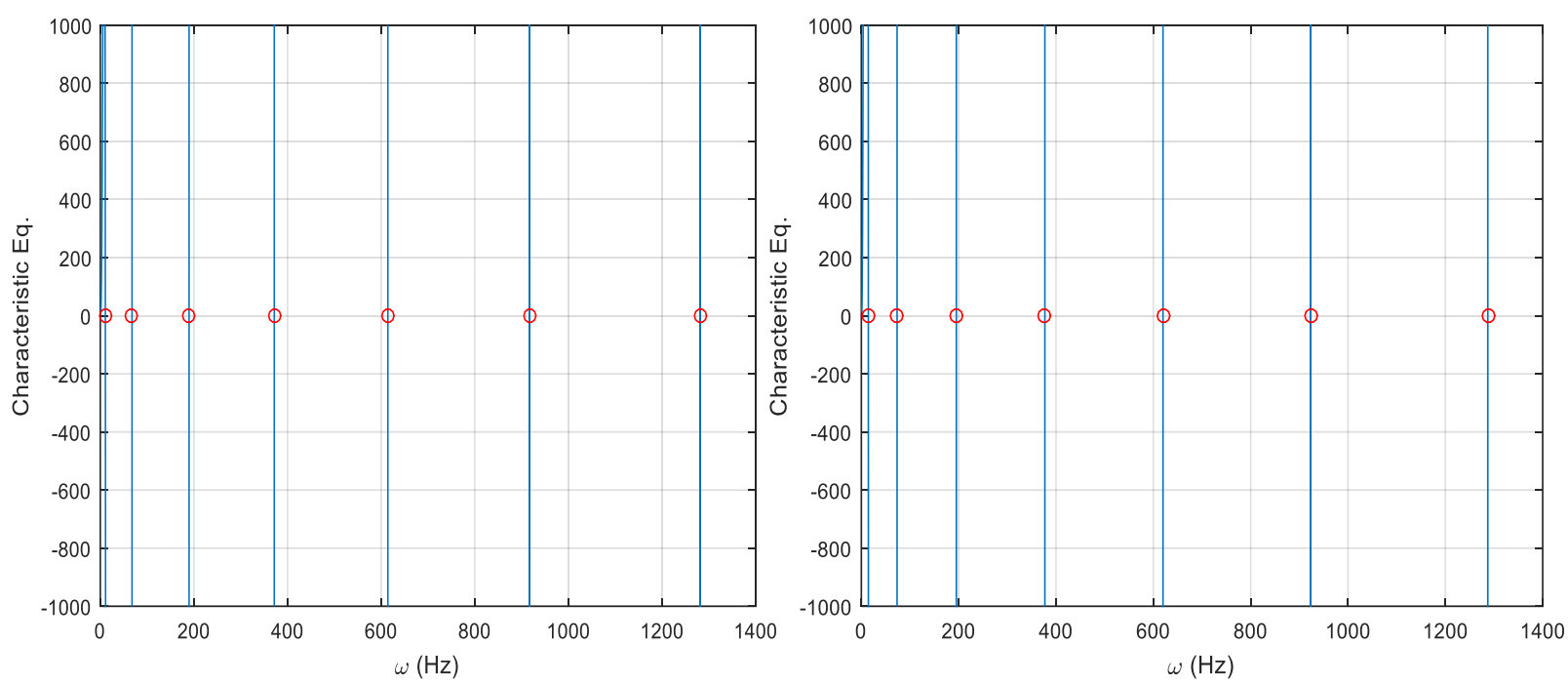

Figure 4. Natural frequencies of uniform and non-uniform cross-section sandwich beams.

Table 2. The first five natural frequencies of uniform cross-section sandwich beams for bending vibarations.

\begin{tabular}{cccc}
\hline $\begin{array}{c}\text { Frequencies } \\
(\mathbf{H z})\end{array}$ & Analytical & $\begin{array}{c}\text { Numerical } \\
\text { (ANSYS) }\end{array}$ & $\begin{array}{c}\text { Difference } \\
\mathbf{( \% )}\end{array}$ \\
\hline $1^{\text {st }}$ Frequency & 10.81 & 10.86 & -0.46 \\
$2^{\text {nd }}$ Frequency & 67.73 & 67.89 & -0.24 \\
$3^{\text {rd }}$ Frequency & 189.60 & 189.38 & 0.12 \\
$4^{\text {th }}$ Frequency & 371.60 & 369.22 & 0.64 \\
$5^{\text {th }}$ Frequency & 614.30 & 606.48 & 1.27 \\
\hline
\end{tabular}

Table 3. The first five natural frequencies of non-uniform cross-section sandwich beams for bending vibarations..

\begin{tabular}{cccc}
\hline $\begin{array}{c}\text { Frequencies } \\
(\mathbf{H z})\end{array}$ & Analytical & $\begin{array}{c}\text { Numerical } \\
\text { (ANSYS) }\end{array}$ & $\begin{array}{c}\text { Difference } \\
\mathbf{( \% )}\end{array}$ \\
\hline $1^{\text {st }}$ Frequency & 14.38 & 14.67 & -2.02 \\
$2^{\text {nd }}$ Frequency & 73.43 & 74.45 & -1.39 \\
$3^{\text {rd }}$ Frequency & 195.20 & 195.50 & -0.15 \\
$4^{\text {th }}$ Frequency & 377.10 & 374.60 & 0.66 \\
$5^{\text {th }}$ Frequency & 619.80 & 610.39 & 1.52 \\
\hline
\end{tabular}




\section{Conclusion}

An analytical solution for vibration analysis of uniform and non-uniform cross-section sandwich cantilever beams is presented. The sandwich beams were considered with small thicknesses and they were handled as Euler-Bernoulli beams for which the shear deformations and the rotational effects are ignored. The natural frequencies of both type sandwich structures were obtained by using the presented analytical solution and ANSYS finite element software. It is observed that the analytical and the numerical results are in good agreement with maximum percentage difference as 1.27 for the uniform cross-section and 1.52 for the non-uniform cross-section sand wich beams. According to obtained results, for dynamic analysis of thin layered sandwich beams the presented solution using the Euler-Bernoulli Beam Theory approach is usefull and can give good results.

\section{References}

[1] Khalili, S.M.R., Nemati, N., Malekzadeh, K., Damanpack, A.R. (2010). Free vibration analysis of sandwich beams using improved dynamic stiffness method. Composite Structures 92, 387-394.

[2] Hamed, E., Rabinovitch, O. (2009). Modeling and dynamics of sandwich beams with a viscoelastic soft core. AIAA Journal 47, 2194-2211.

[3] Palmeri, A., Ntotsios, E. (2016). Transverse vibrations of viscoelastic sandwich beams via galerkinbased state-space approach. Journal of Engineering Mechanics 142, 1-12.

[4] Irazu, L., Elejabarrieta, M.J., Garces, Y. (2015). Dynamic properties of thin sandwich structures: Influence of viscoelastic core. 22nd International Congress on Sound and Vibration, ICSV 2015.

[5] Sakiyama, T., Matsuda, H., Morita, C. (1996). Free vibration analysis of continuous sandwich beams with elastic or viscoelastic cores by applying the discrete Green function. Journal of Sound and Vibration 198, 439-454.

[6] Wang, G., Veeramani, S., Wereley, N.M. (2000). Analysis of sandwich plates with isotropic face plates and a viscoelastic core. Journal of Vibration and Acoustics, Transactions of the ASME 122, 305-312.

[7] Zghal, S., Bouazizi, M.L., Bouhaddi, N. (2014). Reduced-order model for non-linear dynamic analysis of viscoelastic sandwich structures in time domain. MATEC Web of Conferences 16, 1-4.

[8] Barbieri, N., Barbieri, R., Winikes, L.C. (2010). Parameters estimation of sandwich beam model with rigid polyurethane foam core. Mechanical Systems and Signal Processing 24, 406-415.

[9] Benjeddou, A., Guerich, M. (2019). Free vibration of actual aircraft and spacecraft hexagonal honeycomb sandwich panels: A practical detailed FE approach. Advances in Aircraft and Spacecraft Science 6, 169-187.

[10] Lashin, M.M.A., Okasha El-Nady, A. (2015). Free Vibration Analysis of Sandwich Beam Structure Using Finite Element Approach. IOSR Journal of Mechanical and Civil Engineering Ver. I 12, 2278-1684.

[11] Huang, Z., Qin, Z., Chu, F. (2016). Vibration and damping characteristics of sandwich plates with viscoelastic core. Journal of Vibration and Control 22, 1876-1888. 
[12] Cortés, F., Sarriá, I. (2015). Dynamic analysis of three-layer sandwich beams with thick viscoelastic damping core for finite element applications. Shock and Vibration 2015, 1-9.

[13] Zhen, W., Wanji, C. (2018). Free and forced vibration of laminated composite beams. AIAA Journal 56, 2877-2886.

[14] Pham, V.N., Nguyen, D.K., Gan, B.S. (2019). Vibration Analysis of Two-Directional Functionally Graded Sandwich Beams Using a Shear Deformable Finite Element Formulation. Advances in Technology Innovation 4, 152-164.

[15] Kant, T., Swaminathan, K. (2001). Analytical solutions for free vibration of laminated composite and sandwich plates based on a higher-order refined theory. Composite Structures 53, 73-85.

[16] Arvin, H. (2014). Frequency response analysis of higher order composite sandwich beams with viscoelastic core. Iranian Journal of Science and Technology - Transactions of Mechanical Engineering 38, 143-155.

[17] Nasihatgozar, M., Khalili, S.M.R. (2017). Free vibration of a thick sandwich plate using higher order shear deformation theory and DQM for different boundary conditions. Journal of Applied and Computational Mechanics 3, 16-24.

[18] Rajesh, C., Suresh Kumar, J. (2016). Free Vibration Analysis of various Viscoelastic Sandwich Beams. Indian Journal of Science and Technology 9, 1-8.

[19] Abdel Salam, M., Bondok, N.E. (2010). Free Vibration Characteristics for Different Configurations of Sandwitch Beams. International Journal of Mechanical and Mechanics Engineering 10, 41-54.

[20] Purushothaman, V., Guruprasad, V. (2018). Vibration Analysis of Sandwich Beam with Different Core Patterns. International Journal of Engineering Research \& Technology 6, 2-5.

[21] Abdel Salam, M., Abd Raboo, S.M., Bondok, N.E., Sayed, E.K. (2013). An Investigation Into Static and Dynamic Characteristics Of Sandwich Beam. Journal of Applied and Industrial Sciences $1,54-65$.

[22] Dai, G., Zhang, W. (2009). Cell size effects for vibration analysis and design of sandwich beams. Acta Mechanica Sinica 25, 353-365.

[23] Thamburaj, P., Sun, J.Q. (2001). Effect of material and geometry on the sound and vibration transmission across a sandwich beam. Journal of Vibration and Acoustics, Transactions of the ASME 123, 205-212.

[24] Çallioğlu, H., Demir, E., Yilmaz, Y., Sayer, M. (2013). Vibration analysis of functionally graded sandwich beam with variable cross-section. Mathematical and Computational Applications $18,351-360$.

[25] Garooschi, M., Barati, F. (2016). Free Vibration Analysis of Sandwich Cylindrical Panel with Functionally Graded Core by Using ABAQUS Software. Journal of Engineering and Applied Sciences 11, 920-929. 
[26] Kurpa, L., Shmatko, T., Awrejcewicz, J. (2019). Vibration analysis of laminated functionally graded shallow shells with clamped cutout of the complex form by the Ritz method and the Rfunctions theory. Latin American Journal of Solids and Structures 16, 1-16.

[27] Di Sciuva, M., Sorrenti, M. (2019). Bending and free vibration analysis of functionally graded sandwich plates: An assessment of the Refined Zigzag Theory. Journal of Sandwich Structures and Materials 0, 1-43.

[28] Ebrahimi, F., Farazmandnia, N. (2018). Vibration analysis of functionally graded carbon nanotube-reinforced composite sandwich beams in thermal environment. Advances in Aircraft and Spacecraft Science 5, 107-128.

[29] Burlayenko, V.N., Sadowski, T. (2019). Free vibrations and static analysis of functionally graded sandwich plates with three-dimensional finite elements. Meccanica 7.

[30] Ramirez, D.A., Cuba, L.M., Mantari, J.L., Arciniega, R.A. (2019). Bending and free vibration analysis of functionally graded plates via optimized non-polynomial higher order theories. Journal of Applied and Computational Mechanics 5, 281-298.

[31] Zenkour, A.M., Abbas, I.A. (2014). 1371. Free vibration analysis of doubly convex/concave functionally graded sandwich beams. Journal of Vibroengineering 16, 2747-2755.

[32] Li, B., Dong, L., Zhu, L., Chen, X. (2015). On the natural frequency and vibration mode of composite beam with non-uniform cross-section. Journal of Vibroengineering 17, 2491-2502.

[33] Tahouneh, V. (2018). Three-dimensional elasticity solution for vibrational analysis of thick continuously graded sandwich plates with different boundary conditions using a two-parameter micromechanical model for agglomeration. Mechanics of Advanced Composite Structures 5, 4966.

[34] Burlayenko, V.N., Sadowski, T., Dimitrova, S. (2019). Three-Dimensional Free Vibration Analysis of Thermally Loaded FGM Sandwich Plates. Materials 12, 1-20.

[35] Wattanasakulpong, N., Ungbhakorn, V. (2012). Free Vibration Analysis of Functionally Graded Beams with General Elastically End Constraints by DTM. World Journal of Mechanics 02, 297 310.

[36] Yang, X.D., Zhang, W., Chen, L.Q. (2013). Transverse vibrations and stability of axially traveling sandwich beam with soft core. Journal of Vibration and Acoustics, Transactions of the ASME 135, 1-5.

[37] Lougou, K.G., Boudaoud, H., Daya, E.M., Azrar, L. (2016). Vibration modeling of large repetitive sandwich structures with viscoelastic core. Mechanics of Advanced Materials and Structures 23, 458-466. 